\title{
Proposal for Interaction Techniques for Intuitive Virtual Objects Manipulation in Augmented Reality
}

\author{
Takahiro Ishizu, Makoto Sakamoto*, Takaaki Toyota, Masamichi Hori, Satoshi Ikeda and Amane Takei \\ Faculty of Engineering, University of Miyazaki, \\ Miyazaki-City, Miyazaki, Japan
}

Takao Ito

Graduate School of Engineering, Hiroshima University, Hiroshima-City, Hiroshima, Japan

Yu-an Zhang

Department of Computer Technology and Applications, Qinghai University, China

E-mail: hm14002@student.miyazaki-u.ac.jp,fruits2000jp@yahoo.co.jp

\author{
*Corresponding Author \\ E-mail:sakamoto@cs.miyazaki-u.ac.jp \\ http://www.miyazaki-u.ac.jp/
}

\begin{abstract}
Recently, studies for realizing interaction between users and virtual objects using Augmented Reality (AR) have been actively conducted. However, few previous studies have realized that a user intuitively manipulates virtual objects like clay with their bare hands. In this study, we aim at user's intuitive virtual objects manipulation by realizing basic manipulation on virtual objects with their bare hands. In addition, it is considered that the user feels uncomfortable in the manipulation of the virtual objects because of the occlusion problem in AR, and cannot perform an intuitive manipulation. Therefore, in this study, we solve this problem by performing appropriate hidden surface processing within the range of the user's fingertips.
\end{abstract}

Keywords: augmented reality, intuitive manipulation, virtual objects, gesture interaction, occlusion problem.

\section{Introduction}

Augmented reality (AR) is a technique of superimposing information generated by a computer on perceptual information that we receive from real space.

In recent years, technique for realizing interaction between a user and virtual objects in AR has attracted attention.

In interaction technology in $\mathrm{AR}$, it is necessary for the user to be able to intuitively manipulate the virtual objects.

Since the main means of the interaction with the objects in real life is hand, it should also be possible for hand to manipulate virtual objects in the virtual space for intuitive manipulation [1].

Kato et al. [2] introduced soft body virtual objects by using a physics engine, reflected the action of crushing on the virtual object with the user's bare hand in the virtual space, and realized the deformation manipulation of virtual objects by interaction with the user.

Suzuki et al. [3] realized a move manipulation of rigid body virtual objects by interaction with the user.

However, it was not realized that after deforming the virtual object, moving or join the virtual object while maintaining that shape.

(C) The 2019 International Conference on Artificial Life and Robotics (ICAROB2019), Jan. 10-13, B-Con Plaza, Beppu, Oita, Japan 
In this study, we realize to intuitively perform user's manipulation such as deformation of virtual object, maintenance of shape after deformation, joining, and so on, aiming for more advanced interaction between a user and virtual objects.

We believe that this study is useful, for example, by applying this study to modeling work of 3DCG, so that it can be more intuitively.

\section{Proposal}

In this paper, we propose a method to create new virtual objects and a method to distinguish whether the user wants to grasp or wants to move the virtual object. In this way, we realize that the user creates a virtual object, deforms it, moves it while maintaining the deformed state, and joins the two virtual objects.

Moreover, we perform a hidden surface processing to allow the user to recognize the context of the user's hand and the virtual objects.

\subsection{System Component}

We make it possible to realize the interaction of the user and the virtual objects by adopting the Leap Motion Controller specialized for acquisition of threedimensional coordinates of fingers and introducing soft body virtual objects.

This system consists of a Web camera for real image acquisition and marker recognition, the Leap Motion Controller for obtaining three-dimensional coordinates of fingers, and PC for performing arithmetic processing and video output. In this system, we use network programming to prevent interference between the Leap Motion Controller and the Web camera [4]. In this system, we perform acquisition processing of three-dimensional coordinates of fingers with the Leap Motion Controller on the server side. And we perform acquisition processing of image with the Web camera, physical calculation, and video output on the client side.

As shown in the Fig.1, we place the paper on which the AR marker is printed and the Leap Motion Controller, and observe the paper with the Web camera. The virtual objects are displayed by recognizing the AR marker. In this system, we use virtual objects that can be physically computed using the physics engine (Bullet Physics) [5].

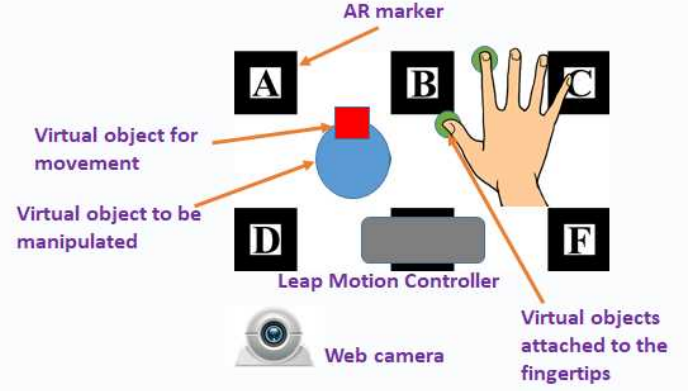

Fig.1. System Component

\section{Hidden Surface Processing}

In AR, a virtual object superimposed on the image of the real space later. For this reason, even when the virtual object is located deeper than the real object, the virtual object is not hidden by the real object, and it is displayed as if the object is always in front. This is called an occlusion problem in $\mathrm{AR}$, and when the user manipulates the virtual object, the user cannot recognize the anteroposterior relationship between the virtual object and the user's hand correctly [6]. For this reason, we think that the user feels uncomfortable visually and cannot perform an intuitive manipulation. Therefore, in this paper, we solve this problem by hidden surface processing of hands and the virtual object in the virtual space, using three-dimensional coordinates of fingers obtained with the Leap Motion Controller and recognizing hand region from skin color region in RGB image obtained with Web camera

\section{Manipulation of Virtual Object}

In this section, we will explain the manipulation of virtual objects by bare hand to realize more intuitive interaction between a user and virtual objects.

\subsection{Generation of Virtual Object}

The user can newly generate a "virtual object to be manipulated", which size is desired by user by gesture of index fingers of both hands (Fig.2).

The procedure will be described below. 
(i) This system acquires the three-dimensional coordinates by putting the user's index finger of the right hand on the Leap Motion Controller.

(ii) This system acquires the three-dimensional coordinates by putting the user's left index finger on the Leap Motion Controller while keeping the state of Step 1.

(iii) The user away the own left index finger with the Leap Motion Controller.

(iv) This system determines the size of the "virtual object to be manipulated" based on the value when the index finger of both hands comes closest and generate the object.

\subsection{Deformation and Moment of The Virtual Object}

It is difficult to distinguish whether the user wants to grasp or wants to move the virtual object. Therefore, this system performs deformation of the virtual object when the user's palm is downwards, and movement of the virtual object when the user's palm is upward. This system decides that the palm is upward when the user's little finger, ring finger, middle finger, index finger, thumb line up from the left of the Leap Motion Controller.

In this system, deformation and movement manipulations on virtual object are performed with only the right hand.

The user turns the palm downwards and crush the "virtual object to be manipulated" with the thumb and index finger, whereby the user's "virtual objects attached to the fingertips" and "virtual object to be manipulated" collide with each other, and the "virtual object to be manipulated" can be deformed (Fig.3).

The user can move "virtual object to for movement" which hold the representative coordinate of "virtual object to be manipulated" by turning the user's palm upwards and moving his/her hand near the virtual object (Fig.4). Since "virtual object for movement" is attached to "virtual object to be manipulated" in advance, "virtual object to be manipulated" can be moved by moving "virtual object for movement".

In the case where two virtual objects are generated, this system obtains the Euclidean distance between the center of the hand and each "virtual object for movement" of each of the two virtual objects and selects a closer "virtual object for movement" as the moving target.

\subsection{Joining of The Virtual Objects}

The user prepares two virtual objects in advance. The user brings one "virtual object to be manipulated" closer to the other "virtual object to be manipulated".

Since collision determination between "virtual object to be manipulated" is not performed in this system, when two "virtual object to be manipulated" continue closer to each other, they eventually overlap each other. And when reaching a fixed distance, the two virtual objects join together (Fig.5).

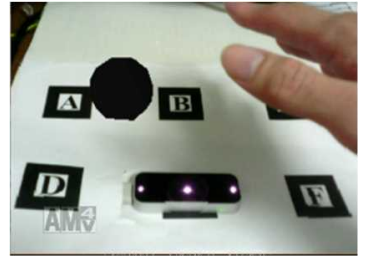

Fig.2. Create Object

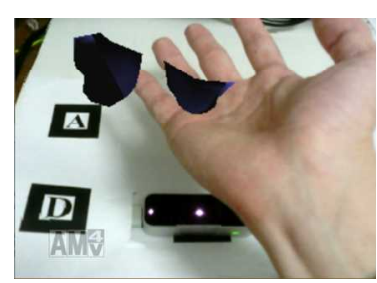

Fig.4. Move Object

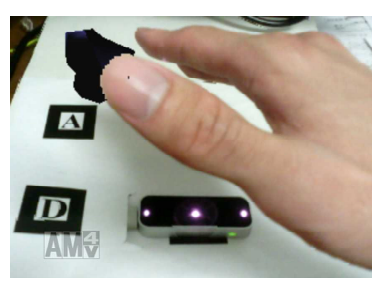

Fig.3. Deform Object

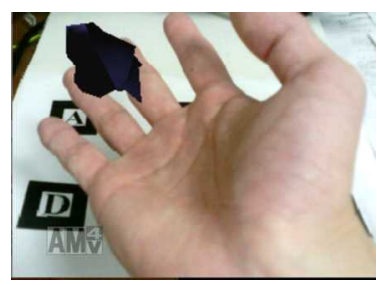

Fig.5. Join Objects

\section{Evaluation}

We investigated the interaction with the virtual object using this system by questionnaire to evaluate whether it is intuitive or not and to extract this system's improvement point. We asked six university students of subjects to perform basic manipulations on virtual objects shown in Fig. 2 to Fig.5.

\subsection{Experiment Method}

After having the subject trial this system, we conducted a question form questionnaire on each item of "good point", "difficult point" in manipulation, and "improvement point" in this system, and we evaluated this system.

\subsection{Experiment Result}

The questionnaire result was as follows.

(C) The 2019 International Conference on Artificial Life and Robotics (ICAROB2019), Jan. 10-13, B-Con Plaza, Beppu, Oita, Japan 
As for "good point", there was some opinions such as "manipulation method is intuitive and easy to understand", and "movements of the hands was reflected in the system".

As for "difficult point", there are some opinions such as "it is difficult to join two virtual objects", "it is difficult to grasp the sense of distance with the virtual object", and "when the hand is manipulated at a low position from the desk, the virtual object does not react in user's gesture".

As for "improvement point", there are some opinions such as "I want feedback when crushing virtual objects", "I want functions that can be transformed into arbitrary figures such as cones and rectangular parallelepipeds by gesture" and "I want a manipulation function to cut off virtual objects".

\section{Consideration}

First, we consider "good point". From the "good point", we found that the interaction manipulation with the virtual object incorporating the gesture is intuitive. Therefore, we believe that it is possible to superimpose the physical phenomenon such as deformation object by hand in the real space on the virtual objects.

Next, we consider "difficult point". We think that the reason why the cause of the opinion that "it is difficult to grasp the sense of distance with the virtual object" is that when the user manipulates the virtual object, an error occurs due to a delay in the position information of the finger acquired with the Leap Motion Controller, and the user feels a sense of incompatibility.

We think that the reason why the cause of the opinion that "it is difficult to join two virtual objects" is that in this system, the coupling of virtual objects are joined when the two virtual objects approach within a certain distance, similarly to the reason that "it is difficult to grasp the sense of distance with the virtual object", an error occurs between the position of the finger on the real space and the position of the finger on the virtual space.

We think that the reason why the cause of the opinion that "when the hand is manipulated at a low position from the desk, the virtual object does not react in user's gesture" is that when the hand is in a low position, the hand covers the Leap Motion Controller, and the coordinates of the fingertips cannot be acquired we considered.

\section{Conclusion}

In this study, we realized a more intuitive manipulation between a user and virtual objects by creating new virtual objects and distinguishing whether the user wants to grasp or wants to move the virtual object.

In the evaluation experiment, we asked six subjects to perform the basic manipulation on the virtual objects and conducted a questionnaire survey. As a result of the experiment, it was shown that by using this system, it is possible to intuitively perform the interaction manipulation with the virtual objects.

In our future work, we will address the issues of "difficult point" and add functions pointed out in "improvement point" so that it can more intuitively interact with virtual objects. Furthermore, we think that it is necessary to increase the precision of interaction, such as enabling finer deformation of virtual objects.

\section{References}

1. V. Buchmann, S. Violich, M. Billinghurst and A Cockburn. "FingARtips-Gesture Based Direct Manipulationin in Augmented Reality". Proc. International Conf. on Computer Graphics and Interractive Techniques, pp.212-221, 2004.

2. S. Kato, S. Ishigaki, S. Suzuki and M. Sato. "A Study on Presentation of Softness of Virtual Object in Gripping Motion with Bare". Journal of The Institute of Image Information and Television Engineers, 2017, VoL 71, No. 3, pp.J121-J124.

3. H. Suzuki , S. Suzuki and M. Sato . "Visual Improvement of AR System Focusing on Grasping Motion with Bare Hands". Journal of The Institute of Image Information and Television Engineers, 2014, VoL 68, No. 7, pp. J299-J301.

4. U. Imamura, et al. "The proposal and development of the novel technology with Augmented Reality". Future University Hakodate 2011 System Science Practice Group Report, 2012.

5. F. Fujisawa. "Information media experiment, Application development using physics engine". [Online] http://slis.tsukuba.ac.jp/ fujisawa.makoto.fu/lecture/iml/ (accessed 2018-03-17).

6. K. Reiji and S. Makoto. "Development of A System enabling Display of Gripping the AR Object Using Leap Motion Controller". The 29th Annual Conference of the Japanese Society for Artificial Intelligence, 2015. 